\title{
Effects of metformin treatment on radiotherapy efficacy in patients with cancer and diabetes: a systematic review and meta-analysis
}

This article was published in the following Dove Press journal:

Cancer Management and Research

\author{
Mingyue Rao ${ }^{1-3}$ \\ Chenlin Gao ${ }^{1,2}$ \\ Man Guo² \\ Betty Yuen Kwan Law ${ }^{1,4}$ \\ Yong $\mathrm{Xu}{ }^{1,2}$ \\ 'Faculty of Chinese Medicine, Macau \\ University of Science and Technology, \\ Avenida Wai Long, Taipa, Macau, \\ China; ${ }^{2}$ Department of Endocrinology, \\ ${ }^{3}$ Department of Oncology, Affiliated \\ Hospital of Southwest Medical \\ University, Luzhou, Sichuan 646000 , \\ China; ${ }^{4}$ State Key Laboratory of \\ Quality Research in Chinese Medicine, \\ Macau University of Science and \\ Technology, Avenida Wai Long, Taipa, \\ Macau, China
}

Correspondence: Betty Yuen Kwan Law Faculty of Chinese Medicine, State Key Laboratory of Quality Research in Chinese Medicine (Macau University of Science and Technology), Avenida Wai Long, Taipa, Macau, 999078, China

Tel +853 88972452

Fax +85328825123

Emailyklaw@must.edu.mo

Yong Xu

Department of Endocrinology, Affiliated Hospital of Southwest Medical University, NO.25 Taiping Street, Luzhou, Sichuan

Province, 646000, China

$\mathrm{Tel}+8608303165003$

Fax +8608303165012

Email xywyll@aliyun.com
Purpose: Metformin is a key pharmaceutical for patients with diabetes mellitus (DM). Metformin also can enhance tumor radiosensitivity in vitro and in vivo. Some retrospective cohort studies have indicated that metformin can improve the efficacy of radiotherapy in patients with cancer and DM. The aim of this systematic review was to evaluate the radiotherapy efficacy of metformin in patients with cancer and DM.

Methods: Multiple databases were queried for studies that address the efficacy of metformin in radiotherapy of patients with cancer and DM. Studies were included that involved comparisons of the short-term tumor responses and long-term survival outcomes of these patients who were managed with or without metformin as well as of nondiabetic patients without metformin. The OR and HR with accompanying 95\% CI were assessed in a random effects model. The main endpoints were 2-year and 5-year overall survival (2y-OS and 5y-OS, respectively).

Results: The database search yielded 17 cohort studies that met the inclusion criteria. The results indicated that the tumor response was higher in patients who also were treated with metformin than in those who were not (OR, 0.48; 95\% CI, 0.22-1.07; $P=0.07)$ and nondiabetic (OR, 0.27; 95\% CI, 0.07-0.98; $P=0.05$ ). Moreover, patients who received metformin had survival benefits compared with patients not treated with metformin (2y-OS: OR, 0.48; 95\% CI, 0.29-0.80; $P=0.005$; 5y-OS: OR, 0.38; 95\% CI, 0.25-0.56; $P<0.00001)$. The metformin-related HRs of OS values were not significantly different.

Conclusion: Metformin appears to improve the tumor response to radiotherapy in patients with cancer and DM and partly yield survival benefits. Despite the apparent advantages provided by metformin treatment on $2 \mathrm{y}-\mathrm{OS}$ and $5 \mathrm{y}$-OS, these retrospective data are at risk of bias and should be interpreted with caution.

Keywords: metformin, cancer, diabetes mellitus, radiotherapy, survival

\section{Introduction}

Diabetes mellitus (DM) is a chronic disease with a high incidence worldwide. Many investigators have shown that having DM may increase the risk of colorectal and endometrial cancers and of any cancer mortality. ${ }^{1-3}$ Results of a meta-analysis showed that patients with cancer and DM have a higher all-cause mortality than do those without DM, especially with respect to colorectal cancer, breast cancer, and endometrial cancer. ${ }^{4}$ Current pharmacologic treatments for patients with DM include insulin and metformin to control the level of blood glucose. Metformin is a first-line drug for controlling circulating insulin levels in DM. ${ }^{5,6}$ Over the past decade, researchers have found that metformin has utility for the treatment of conditions other than DM. 
Specifically, a role for metformin in cancer prevention and treatment has been explored. ${ }^{7}$

Epidemiologic findings have demonstrated that the incidence of cancer in patients with DM who received metformin was lower than among nondiabetics or patients with DM who received a drug other than metformin to control blood glucose. ${ }^{1,8}$ Some retrospective studies have shown that patients with DM who are treated with metformin not only have a lower incidence of cancer but also have improved efficacy of cancer treatment. Metformin has been shown to reduce the biochemical recurrence rate and improve the overall survival (OS) rate of patients with DM and prostate or endometrial cancer. ${ }^{9-11}$ These clinical observations have been supported by experiments in vivo and in vitro, confirming that metformin prevents against carcinogenesis and inhibits the proliferation of cancer cells. ${ }^{12,13}$

Radiotherapy is an essential modality that can be implemented at various phases in the cancer treatment process. Metformin has been found to increase radiosensitivity to non-small cell lung cancer in vitro. ${ }^{14}$ Specifically, metformin reduces oxygen consumption and increases oxygenation in tumor cells by directly inhibiting mitochondrial metabo$\operatorname{lism}^{15}$; this mitigates radiation resistance associated with tumor hypoxia. ${ }^{16}$

Only a review and some retrospective cohort studies have involved the effects of concurrent metformin and radiotherapy in the treatment of patients with cancer and $\mathrm{DM}^{17}$; no large randomized controlled trial (RCT) or metaanalysis has directly addressed this topic. Therefore, there is no sufficient evidence to recommend metformin - rather than other hypoglycemic drugs-alongside radiotherapy for patients with cancer and DM. Moreover, it is unclear whether patients on this treatment regimen would experience survival benefits. We conducted a systematic review and meta-analysis of retrospective cohort studies to investigate whether metformin increases the short-term efficacy and OS benefits of radiotherapy.

\section{Methods}

\section{Literature search strategy}

This systematic review and meta-analysis was conducted in accordance with guidelines set forth by the PRISMA. A literature search was performed on PubMed, Embase, Cochrane Library, Science Direct, Web of Science, CINAHL Plus, ClinicalTrials.gov, and China National Knowledge Infrastructure (CNKI) to obtain articles published on or before April 25, 2018. Search terms were "metformin," in combination with "cancer," "diabetes mellitus," "radiotherapy," and "survival." The search strategy was performed by two investigators (MR and CG).

\section{Eligibility criteria and excluded studies}

The following eligibility criteria were applied to the articles: 1) described a population-based cohort study; 2) involved patients in a treatment group who had cancer and DM and received metformin and radiotherapy; 3 ) involved patients in a control group with or without DM, but no control patient received metformin; 4) included outcome measures of qualitative improvement in tumor response and OS; 5) was a high-quality study, based on a Newcastle-Ottawa Scale (NOS) score of $\geq 6$; and 6) was written in English or Chinese. Two reviewers independently assessed the articles based on titles and abstracts and excluded studies that addressed animal models or in vitro experiments, lacked original data, were not related to metformin and radiotherapy, or duplicated a study that had already been recovered from the literature search. After this screen, full-text articles of the studies deemed relevant were retrieved. These articles were reviewed and were excluded from the study if the comparison group did not conform to the inclusion/exclusion criteria. At this stage, studies were excluded that did not present data on efficacy or survival outcomes or that presented inconsistent data. Disagreements about eligibility were resolved by discussion between the authors (MR and CG). If agreement could not be reached, a third arbiter (YX) was consulted.

\section{Data extraction}

The following information was extracted from each included cohort study: author names, publication year, site of original tumor, study groups, specimens collected, radiotherapy dose, concurrent treatment during radiotherapy, use of adjuvant therapy and type, outcomes, and quality score in NOS. Study investigators were asked via email to provide information that had been omitted from the published articles. Information was independently extracted by MR and CG, and all extracted data were confirmed by MG.

\section{Statistical analysis}

For studies that included qualitative short-term curative findings and long-term follow-up in each group, we pooled the ORs in a random effects model to facilitate generalizability of results. For studies that reported quantitative survival outcomes (HR and accompanying in 95\% CI) in comparisons with patients with diabetes on metformin $(\mathrm{D}+\mathrm{M})$ and those with diabetes not on metformin (D-M) or nondiabetic patients not on metformin (N-M), we combined the logHR 
and the standard error (SE) in a random effects model. In the analysis of HRs with $95 \%$ CIs for disease-free survival (DFS), distant metastasis-free survival (DMFS), and OS, HR data were derived from univariate analysis and multivariate analysis. (When both types of data existed, multivariate data were extracted as a priority.) We assessed statistical heterogeneity using $Q$-tests and the $I^{2}$ statistic. All analyses were carried out using Review Manager (RevMan) software, version 5.0 (Cochrane, Copenhagen, Denmark).

\section{Results}

Our search yielded more than 1,370 studies for initial review. After screening titles and abstracts, 30 articles remained for full-text review. Thirteen of these articles did not meet inclusion criteria, and the remaining 17 articles were included in a meta-analysis (Figure 1)..$^{18-34}$ The 17 cohort studies involved effect sizes of metformin-enhanced radiotherapy in the following cancers: prostate cancer, four studies; head and neck cancer, four studies; rectal cancer, two studies; lung cancer, three studies; esophageal cancer, three studies; liver cancer, one study. The included studies all had comparisons of $\mathrm{D}-\mathrm{M}$ and/or N-M patient groups and addressed pathologic complete response (pCR), DFS, DMFS, and OS after treatment. Nine of the 17 studies included comparisons of $\mathrm{D}+\mathrm{M}$ and $\mathrm{D}-\mathrm{M}$ in terms of the HR OS/DFS/DMFS and 95\% CI (Table 1). The 17 studies comprise 14,333 patients with cancer who received radiotherapy with or without chemotherapy.

\section{Pathologic complete response}

We analyzed short-term curative effects in terms of the $\mathrm{pCR}$ of $\mathrm{D}+\mathrm{M}$ and $\mathrm{D}-\mathrm{M}$ or $\mathrm{N}-\mathrm{M}$ after radiotherapy. Five articles contained pCR data for patients, all of whom had gastrointestinal tumors. These patients received neoadjuvant concurrent chemoradiotherapy followed by surgery. The pCR data were obtained postoperatively. The data were converted into discontinuation variables, and ORs were pooled in a random effects model to facilitate generalizability of results. Our results indicated that the D+M group appeared to be more likely to improve in postoperative $\mathrm{pCR}$ compared with the D-M group (OR, 0.48; 95\% CI, 0.22-1.07; $P^{2}=51.0 \%$; $P=0.07$ ) or the N-M group (OR, $0.27 ; 95 \% \mathrm{CI}, 0.07-0.98$; $\left.I^{2}=54.0 \% ; P=0.05\right)$ (Figure 2).

\section{Distant metastasis-free survival}

We analyzed long-term survival outcomes in terms of DMFS after radiotherapy in the $\mathrm{D}+\mathrm{M}, \mathrm{D}-\mathrm{M}$, and $\mathrm{N}-\mathrm{M}$ groups. The DMFS comparisons originated from different diseases and were represented as 2-year DMFS (2y-DMFS) and 5-year DMFS (5y-DMFS). The former comprises lung and esophageal cancer, whereas the latter included prostate, head and neck, and esophageal carcinoma. There was no significant difference in $2 \mathrm{y}$-DMFS between the $\mathrm{D}+\mathrm{M}$ and $\mathrm{D}-\mathrm{M}$ groups (OR, $0.54 ; 95 \%$ CI, $0.16-1.81 ; I^{2}=36.0 \% ; P=0.32$ ) or in the 5y-DMFS (D+M vs D-M OR, 0.53; 95\% CI, 0.15-1.85; $I^{2}=75.0 \% ; P=0.32$; and $\mathrm{D}+\mathrm{M}$ vs N-M OR, $1.29 ; 95 \% \mathrm{CI}$, $0.72-2.31 ; I^{2}=41.0 \% ; P=0.39$ ) (Figure 3 ).

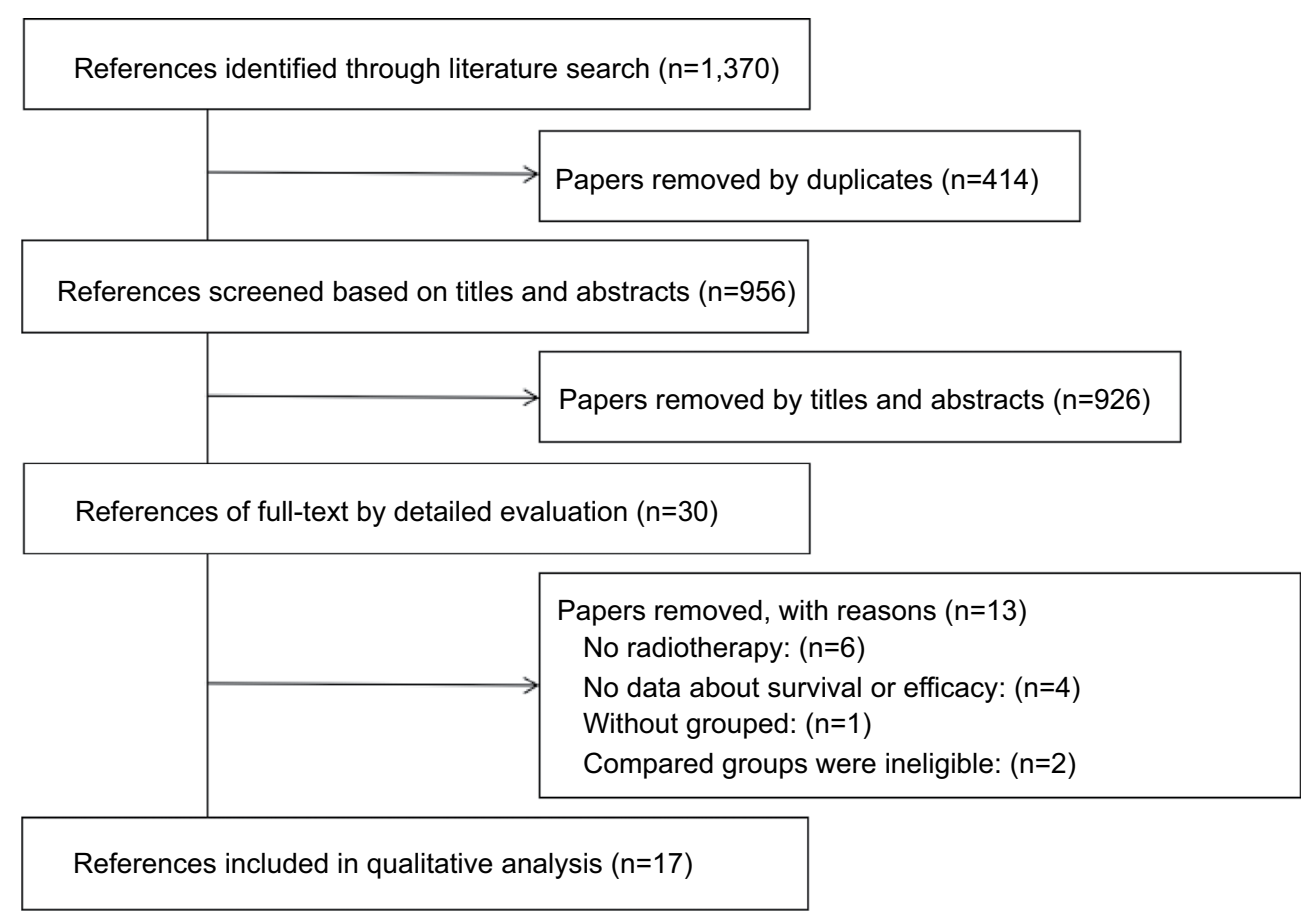

Figure I Flow diagram showing study selection. 


\begin{tabular}{|c|c|c|c|c|c|c|c|c|c|c|c|c|c|}
\hline 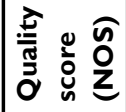 & $n$ & $\infty$ & 0 & $\infty$ & $\infty$ & $n$ & & 6 & $\wedge$ & $\Lambda$ & 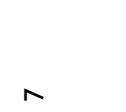 & & $n$ \\
\hline 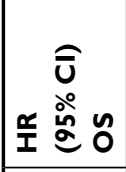 & \begin{tabular}{l}
0 \\
\multirow{0}{0}{} \\
$z$
\end{tabular} & 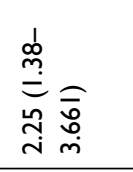 & $\stackrel{0}{0}$ & $\begin{array}{l}0 \\
\text { Z } \\
\text { z }\end{array}$ & 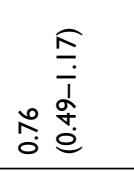 & $\stackrel{0}{0}$ & & $\begin{array}{l}0 \\
0 \\
\text { Zू } \\
\end{array}$ & 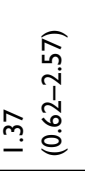 & 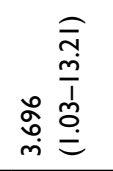 & $\stackrel{0}{0}$ & & 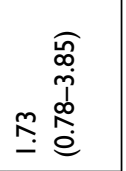 \\
\hline 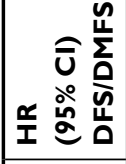 & $\begin{array}{l}0 \\
\vdots \\
\text { z } \\
\end{array}$ & 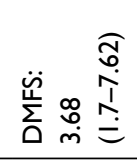 & $\begin{array}{l}0 \\
\check{0} \\
z\end{array}$ & $\begin{array}{l}0 \\
\tilde{0} \\
z\end{array}$ & 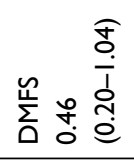 & $\stackrel{0}{0}$ & & $\begin{array}{lll}0 \\
\vdots \\
z\end{array}$ & $\begin{array}{l}\stackrel{0}{0} \\
\text { Ż }\end{array}$ & $\stackrel{0}{0}$ & 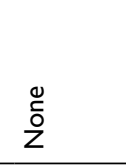 & & 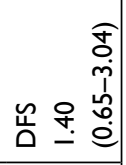 \\
\hline 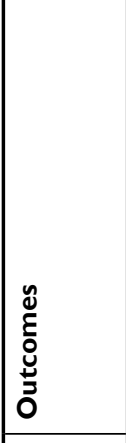 & 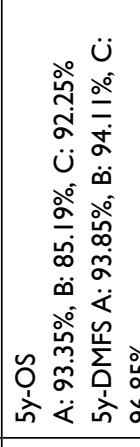 & 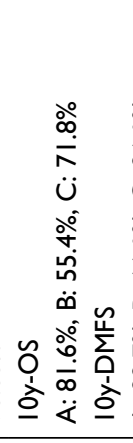 & 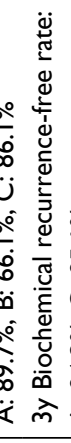 & 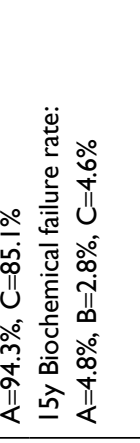 & 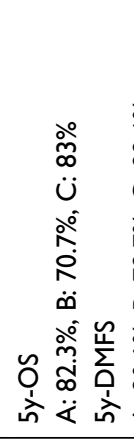 & 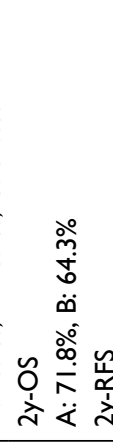 & 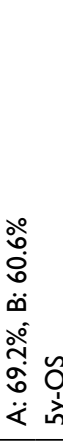 & 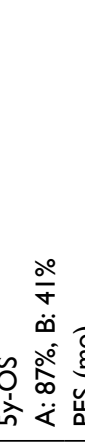 & 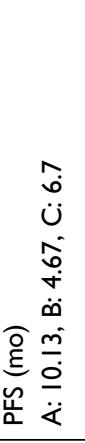 & 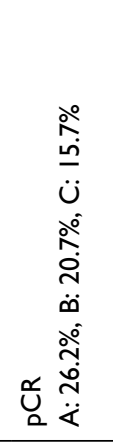 & 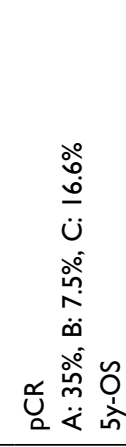 & 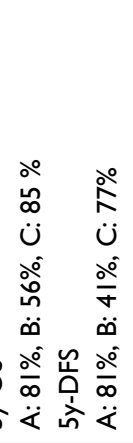 & 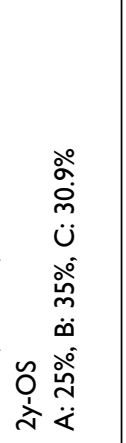 \\
\hline 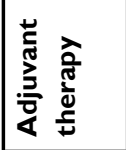 & 占 & $\stackrel{0}{\stackrel{0}{0}}$ & $\begin{array}{l}0 \\
\stackrel{0}{0} \\
z\end{array}$ & 占 & $\begin{array}{l}0 \\
\stackrel{0}{0} \\
z\end{array}$ & $\stackrel{0}{\tilde{0}}$ & & $\begin{array}{lll}0 \\
\vdots \\
z\end{array}$ & 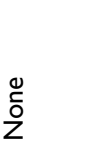 & 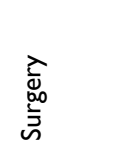 & 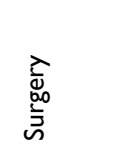 & & $\frac{0}{\tilde{0}}$ \\
\hline 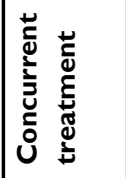 & 占 & $\begin{array}{l}0 \\
\stackrel{0}{z}\end{array}$ & $\begin{array}{l}0 \\
\stackrel{0}{0} \\
z\end{array}$ & 占 & 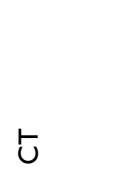 & 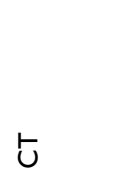 & & 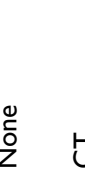 & $\overleftarrow{u}$ & ๖ & $\mathfrak{u}$ & & $\mathfrak{u}$ \\
\hline 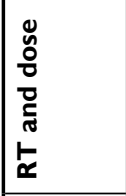 & 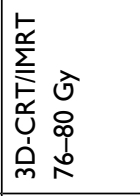 & $\stackrel{\vdash}{\propto}$ & $\vdash$ & 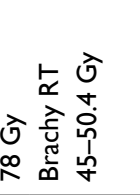 & $\begin{array}{l}\text { 交 } \\
\underline{\underline{\Sigma}}\end{array}$ & 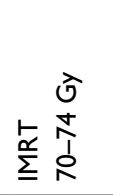 & & $\frac{1}{\alpha}$ & 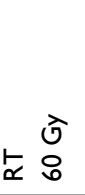 & 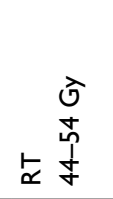 & 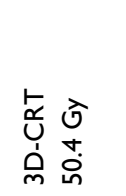 & & 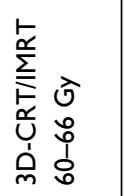 \\
\hline 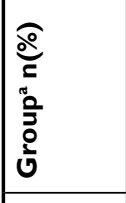 & 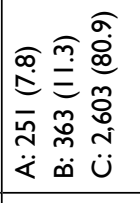 & 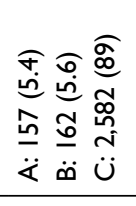 & 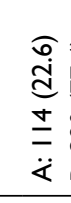 & 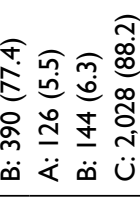 & 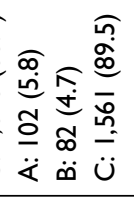 & 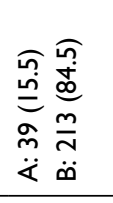 & & 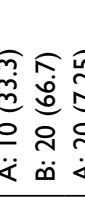 & 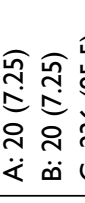 & 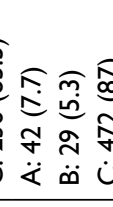 & 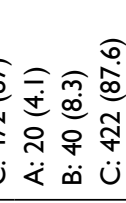 & & 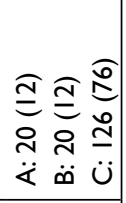 \\
\hline 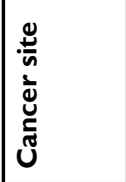 & 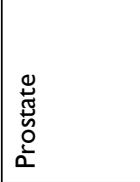 & 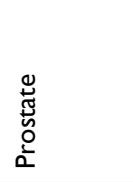 & 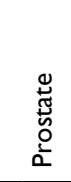 & 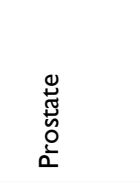 & 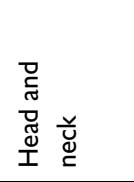 & 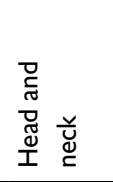 & & 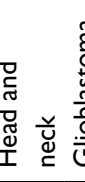 & 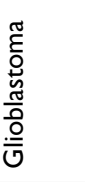 & 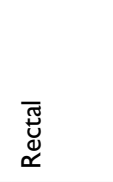 & 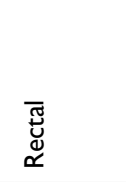 & & $\stackrel{\infty}{\mathfrak{S}^{3}}$ \\
\hline 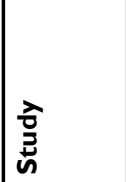 & 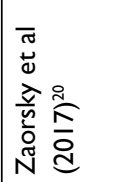 & 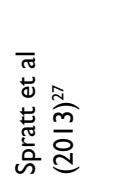 & 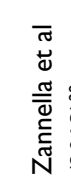 & 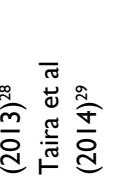 & 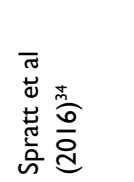 & 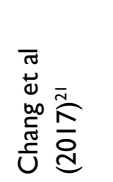 & & 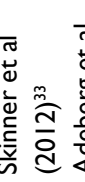 & 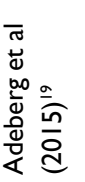 & 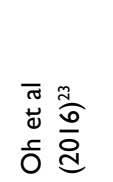 & 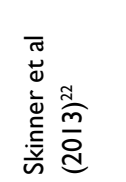 & & 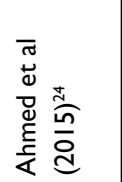 \\
\hline
\end{tabular}




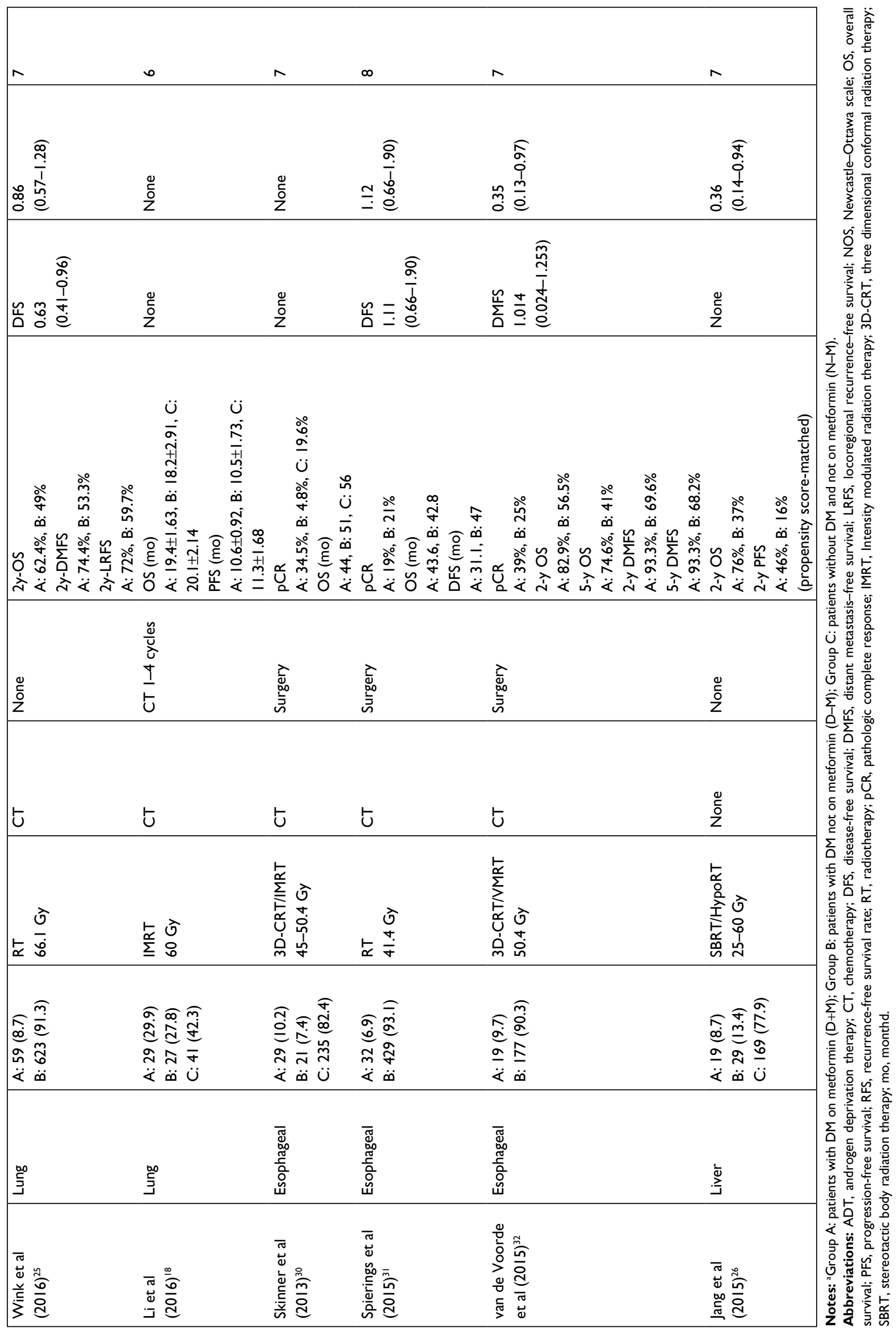




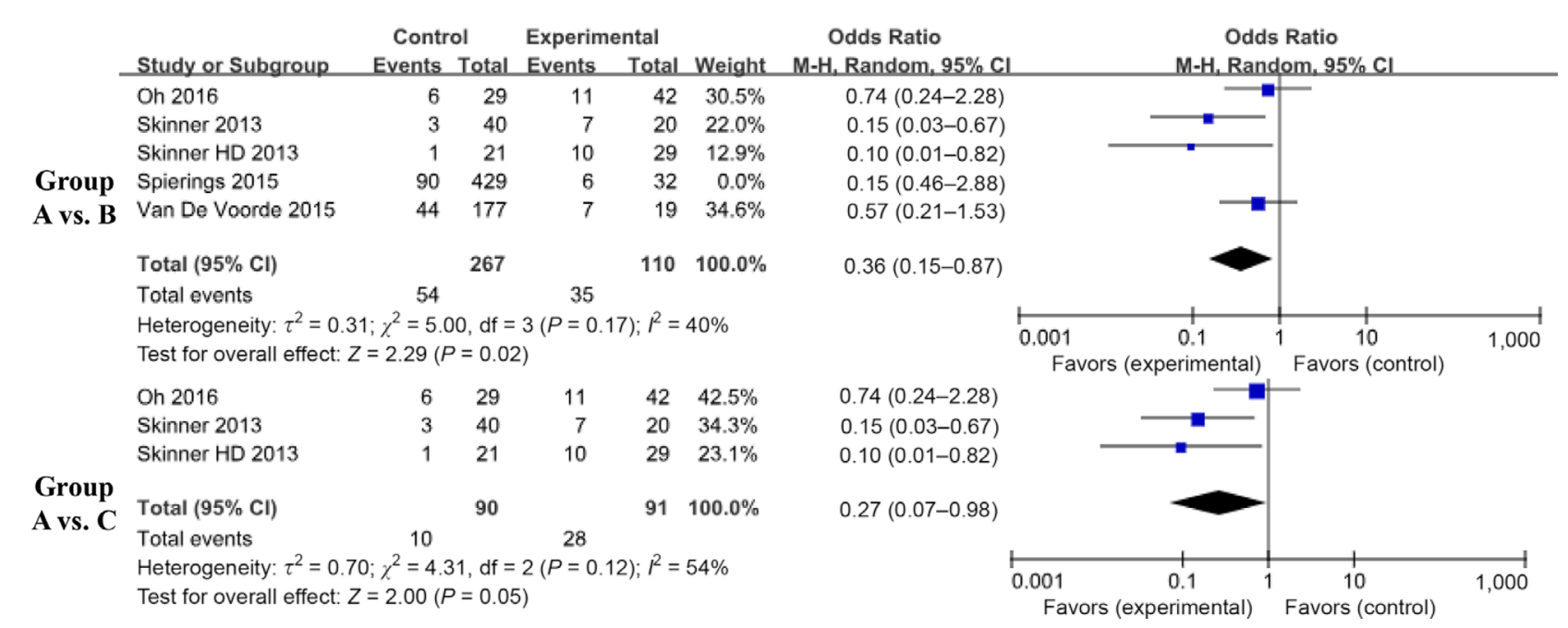

Figure 2 Findings of a meta-analysis of studies with discontinuation data on improvement in pathologic complete response for group $A$ vs $B$ and $C$, in terms of estimated ORs and $95 \%$ Cls.

Notes: Group A: patients with DM on metformin (D+M); Group B: patients with DM not on metformin (D-M); Group C: patients without DM and not on metformin (N-M). Abbreviation: DM, diabetes mellitus.

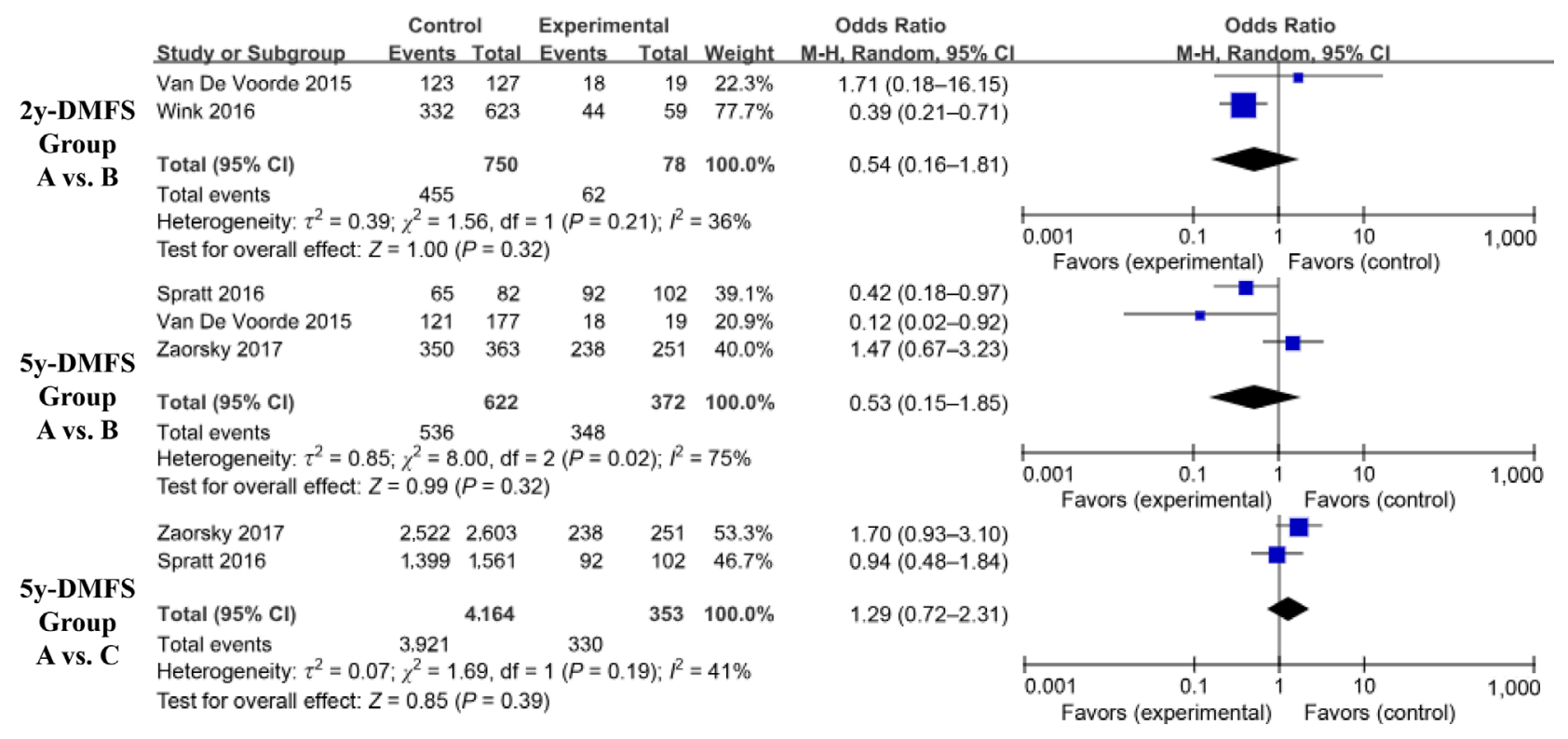

Figure 3 Findings of a meta-analysis of studies with discontinuation data on improvement in 2y-or 5y-DMFS in group A vs B and C, with estimated ORs and $95 \%$ Cls. Notes: Group A: patients with DM on metformin (D+M); Group B: patients with DM not on metformin (D-M); Group C: patients without DM and not on metformin (N-M). Abbreviations: DM, diabetes mellitus; DMFS, distant metastasis-free survival.

\section{Overall survival}

We compared OS results - in terms of 2y-OS and 5y-OS - for patients with various cancer types. The $2 \mathrm{y}$-OS was composed of head and neck, lung, and liver cancer. The 5y-OS involved prostate, head and neck, rectal, and esophageal carcinoma. There was no significant improvement in the $5 \mathrm{y}-\mathrm{OS}$ for $\mathrm{D}+\mathrm{M}$ group vs the $\mathrm{N}-\mathrm{M}$ group (OR, 0.64; 95\% CI, 0.64-1.32; $P=0 \% ; P=0.64)$. The $2 \mathrm{y}-\mathrm{OS}$ and $5 \mathrm{y}-\mathrm{OS}$ in the $\mathrm{D}+\mathrm{M}$ group were higher than in the D-M group (2y-OS: OR, $0.48 ; 95 \%$ CI, $0.29-0.8 ; P^{2}=33.0 \% ; P=0.005$ and $5 \mathrm{y}-\mathrm{OS}: \mathrm{OR}, 0.38 ; 95 \%$ CI, $0.25-0.56 ; I^{2}=0 \% ; P<0.00001$ ) (Figure 4).

\section{HR for DFS, DMFS, and OS in the D+M group}

Pooled HRs for the DFS and DMFS for patients in the D+M group are presented in Table 1 and in Figure 5. Overall, the 


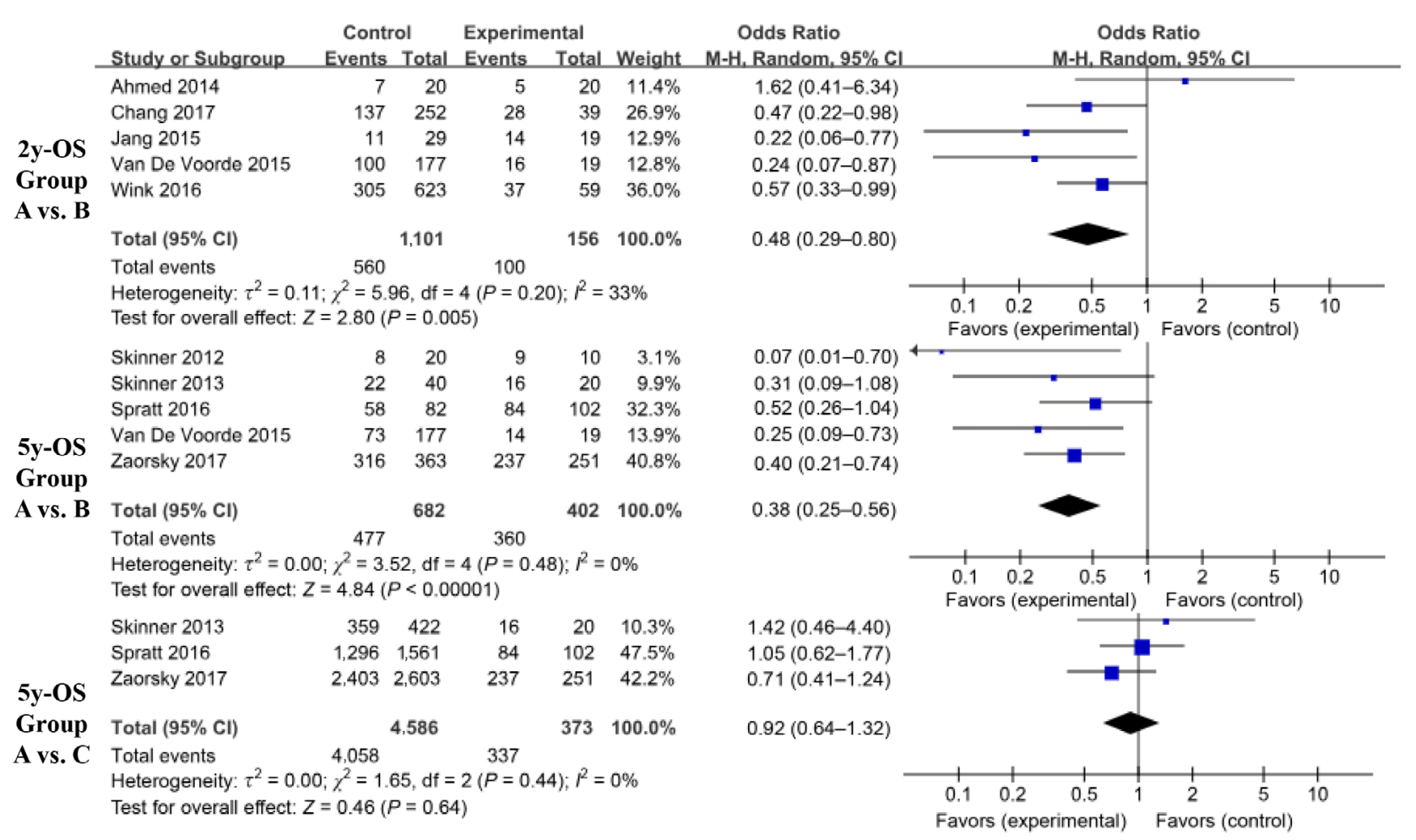

Figure 4 Findings of a meta-analysis of studies with dichotomous data on improvement in $2 y$ - or $5 y$-OS in group A vs B and C, with estimated ORs and $95 \%$ Cls.

Notes: Group A: patients with DM on metformin (D+M); Group B: patients with DM not on metformin (D-M); Group C: patients without DM and not on metformin (N-M). Abbreviation: DM, diabetes mellitus.

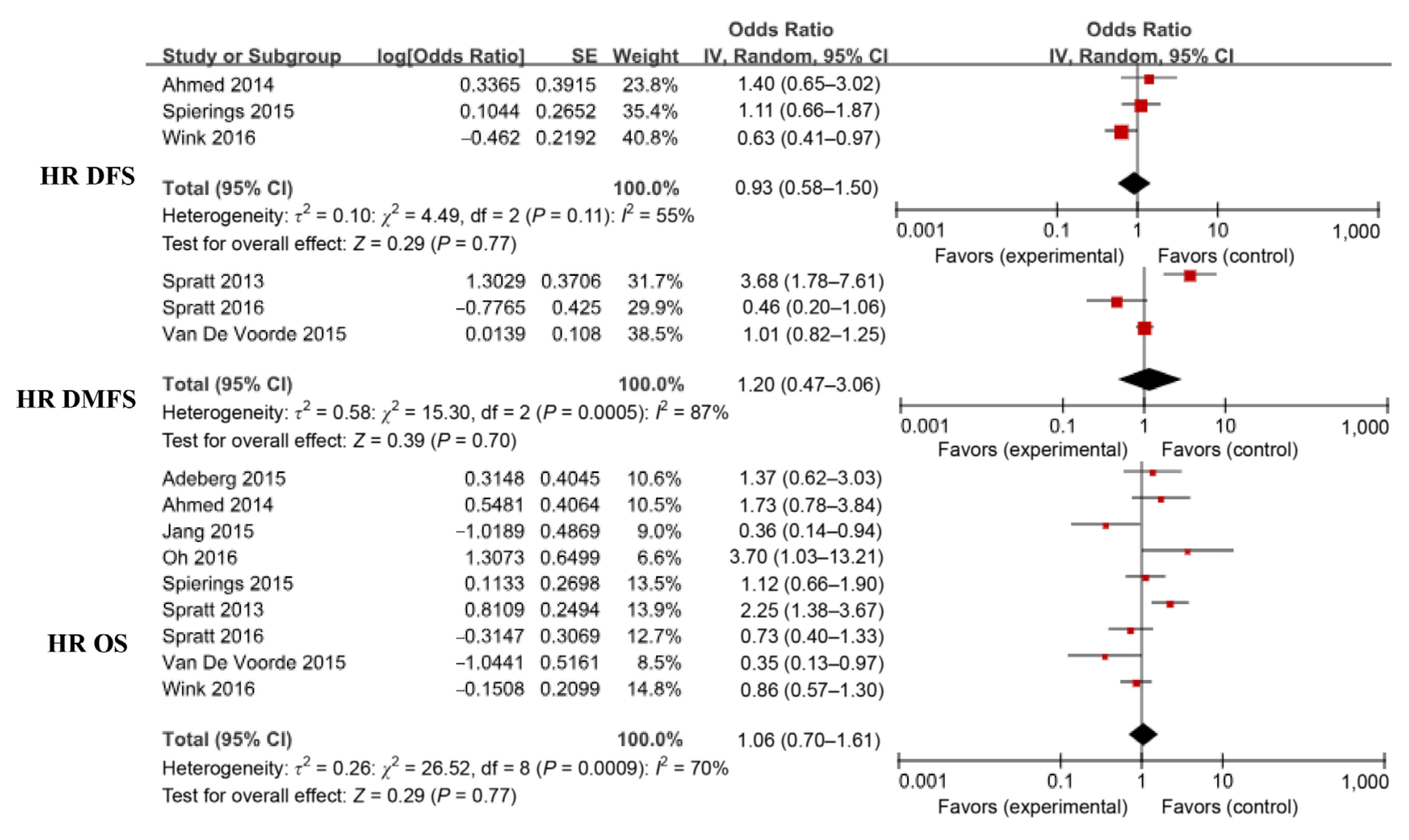

Figure 5 Meta-analysis results of the effect of metformin use on survival outcomes (DFS, DMFS, and OS) in patients with cancer and DM who also received radiotherapy. Abbreviations: DM, diabetes mellitus; DFS, disease-free survival; DMFS, distant metastasis-free survival; OS, overall survival. 
results showed that the use of metformin did not produce a significantly decreased risk of progression or metastasis in patients with DM and cancer who underwent radiotherapy (DFS: HR, 0.93; 95\% CI, 0.58-1.50; DMFS: HR, 1.2; 95\% CI, 0.47-3.06) under the random effects model. This finding can be attributed, at least in part, to heterogeneity among the 17 studies.

To examine the effect of metformin management on OS, nine of the 17 studies were pooled into a meta-analysis. The findings indicated a nonsignificantly reduced risk of death for patients treated with metformin (HR, 1.06; 95\% CI, 0.7-1.61; $I^{2}=70 \% ; P=0.77$ ). Because the number of studies involved in the comparison of indicators was relatively small, we did not perform the Egger test of publication bias.

\section{Discussion}

Metformin is an oral medication that controls blood glucose. Its safety, efficacy, and low cost make it an attractive first-line agent in the treatment of type 2 diabetes and other insulin resistance-related diseases. ${ }^{35}$ At present, the clinical utility of metformin in the treatment of aging and various diseasesincluding cancer, cardiovascular disease, and intestinal dysbacteriosis - has attracted a great deal of attention. ${ }^{36}$ Metformin can reduce the risk of cancer in patients with type 2 diabetes, and a large body of epidemiologic evidence supports the favorable effects of metformin on cancers of the colon, lung, liver, ovary, and breast. ${ }^{37}$ Metformin also can exert a positive influence as an adjuvant in radiotherapy and/or chemotherapy, primarily in patients with lung, ${ }^{38}$ liver, colon, esophageal, or prostate cancer. Other malignancies, including melanoma, bladder, and head and neck cancer, also can be improved by a combination of metformin and antitumor therapeutic approaches. ${ }^{39-41}$

Herein, we describe the results of a systematic review and meta-analysis involving 17 retrospective cohort studies. Our aim was to explore the synergistic effect of metformin on radiotherapy in clinical practice. The phenomenon of increased radiosensitivity in the presence of metformin has been supported by preclinical data. Specifically, metformin combined with radiation was found to enhance DNA damage in vitro, as measured by indices such as phosphorylation of histone protein $\mathrm{H} 2 \mathrm{AX}$ and the olive tail moment. ${ }^{42,43}$

Metformin selectively inhibits complex I; mitochondrial glycerophosphate dehydrogenase may increase the production of reactive oxygen species and decrease glutathione induced by metformin. The result of this process would be to exacerbate DNA damage. The molecular mechanisms that regulate metformin-enhanced radiosensitivity involve p53- and AMPK-mediated signaling pathways. These two signaling pathways have multiple points of cross-talk. Tumor suppressor p53 is a transcription factor that, under genotoxic or metabolic stress, is phosphorylated, stabilized, and activated, thereby inducing cell cycle arrest, apoptosis, or metabolic adaptation. ${ }^{44}$ Skinner et al found that metformin radiosensitization was related to the expression of mutant p53. ${ }^{33}$ However, the role of p53 was potentially site-specific, dependent on certain mutations, and likely to be affected by other genetic changes in cancer. ${ }^{45}$ Results of another study demonstrated that the treatment of cells with siRNA against AMPK could prevent or reduce metformin-mediated radiosensitization. ${ }^{46}$ However, other investigators found that metformin had no significant effect on radiation effects after AMPK gene knockout. Therefore, the role of AMPK in the radiation response in the presence of metformin remains unclear.

To our knowledge, the current study was the first metaanalysis of the potentially synergistic antitumor effects of metformin and radiotherapy on treatment of patients with cancer and DM. An advantage of this study was the inclusion of PubMed, Embase, Cochrane Library, Science Direct, Web of Science, CINAHL Plus, ClinicalTrials.gov, and CNKI databases in the initial literature search. However, because these studies were not randomized and involve disparate patient groups, it is difficult to draw conclusions.

Included patients were categorized as D+M, D-M, or $\mathrm{N}-\mathrm{M}$ in this meta-analysis. Our findings of the short-term curative effects of radiotherapy indicated that the pCR was increased in patients with tumors of the gastrointestinal system who were treated with metformin (rectal cancer and esophageal carcinoma). However, when these nonrandomized data were pooled, we did not observe a significant statistical advantage. In the absence of combined data, the researchers found discordant results in the biochemical failure rate. Specifically, when the 2y-DMFS and 5y-DMFS were compared in cancers of the lung, esophagus, prostate, and head and neck, we found that metformin did not produce a significant improvement in survival outcomes, regardless of whether the comparison was with $\mathrm{D}-\mathrm{M}$ or $\mathrm{N}-\mathrm{M}$. In the comparison of $5 \mathrm{y}-\mathrm{OS}$, the D+M group had no survival advantage over the $\mathrm{N}-\mathrm{M}$ group. When $\mathrm{D}+\mathrm{M}$ and $\mathrm{D}-\mathrm{M}$ groups were compared with respect to the $2 \mathrm{y}-\mathrm{OS}$ and $5 \mathrm{y}-\mathrm{OS}$, metformin use was associated with a survival benefit.

In the present study, the diseases compared included cancers of the lung, liver, esophagus, rectum, prostate, and head and neck. There are inherent differences in biological characteristics among these cancers, and any survival benefit 
of metformin in certain cancers could have been dampened by a lack of effect in other cancers.

Our findings must be interpreted with caution. This meta-analysis was not based on RCTs and involved studies with inherent biases. We also determined that the $\log \mathrm{HR}$ and SE for patients who received radiotherapy and metformin did not show a benefit of OS compared with the D-M group.

This study had several limitations. We only considered the effect of metformin as a radiosensitizer applied in combination with radiotherapy, but for patients with DM, metformin is a lifelong treatment. The possible role of some potential confounders for therapeutic effect of tumor, such as duration of diabetes, duration of metformin exposure, the dose of metformin used, blood glucose level, and the presence or not of insulin resistance, was not strictly assessed in these studies. Only seven studies mentioned the metformin daily doses ranges from 500 to 2,000 mg. Only one study further discussed a significant dose-dependent effect of metformin on response, with doses of greater than $1,500 \mathrm{mg} /$ day, associated with improved pCR. ${ }^{30} \mathrm{In}$ addition, there was no homogeneity among studies in the comprehensive antitumor treatment strategy applied. The authors of the included studies did not provide details on whether patients continued treatment with adjuvant therapy after radiotherapy. From a clinical viewpoint, the current study involved too small of a sample size to make any substantive conclusions. These limitations highlight that RCTs are needed to clarify the impact of metformin on cancer prevention and treatment, particularly in nondiabetic patients with cancer.

\section{Conclusion}

The results of the present meta-analysis indicate inconsistencies in the effect of metformin alongside radiotherapy in patients with cancer and DM. Metformin use seemed to correlate with improved tumor response to treatment, but this effect did not totally translate to survival benefits. Despite the favorable effects of metformin on $2 y-O S$ and $5 y-O S$ in multiple patients with cancer, the retrospective studies reviewed herein had risk of bias. RCTs are needed to delineate the advantages of metformin in these patient groups and to clarify the mechanism by which metformin could enhance radiosensitivity.

\section{Acknowledgment}

The authors would like to thank BioMed Proofreading LLC for English expression polishing.

\section{Disclosure}

The authors report no conflicts of interest in this work.

\section{References}

1. Franciosi M, Lucisano G, Lapice E, Strippoli GF, Pellegrini F, Nicolucci A. Metformin therapy and risk of cancer in patients with type 2 diabetes: systematic review. PLoS One. 2013;8(8):e71583.

2. Larsson SC, Orsini N, Wolk A. Diabetes mellitus and risk of colorectal cancer: a meta-analysis. J Natl Cancer Inst. 2005;97(22):1679-1687.

3. Friberg E, Orsini N, Mantzoros CS, Wolk A. Diabetes mellitus and risk of endometrial cancer: a meta-analysis. Diabetologia. 2007;50(7):1365-1374.

4. Barone BB, Yeh HC, Snyder CF, et al. Long-term all-cause mortality in cancer patients with preexisting diabetes mellitus: a systematic review and meta-analysis. JAMA. 2008;300(23):2754-2764.

5. Dowling RJ, Goodwin PJ, Stambolic V. Understanding the benefit of metformin use in cancer treatment. BMC Med. 2011;9:33.

6. Koritzinsky M. Metformin: a novel biological modifier of tumor response to radiation therapy. Int J Radiat Oncol Biol Phys. 2015;93(2): 454-464.

7. Pollak MN. Investigating metformin for cancer prevention and treatment: the end of the beginning. Cancer Discov. 2012;2(9):778-790.

8. Evans JM, Donnelly LA, Emslie-Smith AM, Alessi DR, Morris AD. Metformin and reduced risk of cancer in diabetic patients. $B M J$. 2005;330(7503):1304-1305.

9. Yu H, Yin L, Jiang X, et al. Effect of metformin on cancer risk and treatment outcome of prostate cancer: a meta-analysis of epidemiological observational studies. PLoS One. 2014;9(12):e116327.

10. Stopsack KH, Ziehr DR, Rider JR, Giovannucci EL. Metformin and prostate cancer mortality: a meta-analysis. Cancer Causes Control. 2016;27(1):105-113.

11. Nevadunsky NS, van Arsdale A, Strickler HD, et al. Metformin use and endometrial cancer survival. Gynecol Oncol. 2014;132(1):236-240.

12. Memmott RM, Mercado JR, Maier CR, Kawabata S, Fox SD, Dennis PA. Metformin prevents tobacco carcinogen — induced lung tumorigenesis. Cancer Prev Res. 2010;3(9):1066-1076.

13. Ben Sahra I, Laurent K, Loubat A, et al. The antidiabetic drug metformin exerts an antitumoral effect in vitro and in vivo through a decrease of cyclin D1 level. Oncogene. 2008;27(25):3576-3586.

14. Storozhuk Y, Hopmans SN, Sanli T, et al. Metformin inhibits growth and enhances radiation response of non-small cell lung cancer (NSCLC) through ATM and AMPK. Br J Cancer. 2013;108(10):2021-2032.

15. Lin A, Maity A. Molecular pathways: a novel approach to targeting hypoxia and improving radiotherapy efficacy via reduction in oxygen demand. Clin Cancer Res. 2015;21(9):1995-2000.

16. Hill RP, Bristow RG, Fyles A, Koritzinsky M, Milosevic M, Wouters BG. Hypoxia and predicting radiation response. Semin Radiat Oncol. 2015;25(4):260-272.

17. Samsuri NAB, Leech M, Marignol L. Metformin and improved treatment outcomes in radiation therapy - a review. Cancer Treat Rev. 2017;55:150-162.

18. Li DM, Wang L, Mu QX, Sun XC. Effect of prognosis of metformin on limited stage small cell lung cancer treated with concurrent chemoradiotherapy. Acta Universitatis Medicinalis Nanjing (Natural Science). 2016;35(6):841-843.

19. Adeberg S, Bernhardt D, Ben Harrabi S, et al. Metformin influences progression in diabetic glioblastoma patients. Strahlenther Onkol. 2015;191(12):928-935.

20. Zaorsky NG, Shaikh T, Ruth K, et al. Prostate cancer patients with unmanaged diabetes or receiving insulin experience inferior outcomes and toxicities after treatment with radiation therapy. Clin Genitourin Cancer. 2017;15(2):326-335.

21. Chang PH, Yeh KY, Wang CH, et al. Impact of metformin on patients with advanced head and neck cancer undergoing concurrent chemoradiotherapy. Head Neck. 2017;39(8):1573-1577. 
22. Skinner HD, Crane $\mathrm{CH}$, Garrett $\mathrm{CR}$, et al. Metformin use and improved response to therapy in rectal cancer. Cancer Med. 2013;2(1):99-107.

23. Oh BY, Park YA, Huh JW, et al. Metformin enhances the response to radiotherapy in diabetic patients with rectal cancer. J Cancer Res Clin Oncol. 2016;142(6):1377-1385.

24. Ahmed I, Ferro A, Cohler A, et al. Impact of metformin use on survival in locally-advanced, inoperable non-small cell lung cancer treated with definitive chemoradiation. J Thorac Dis. 2015;7(3):346-355.

25. Wink KC, Belderbos JS, Dieleman EM, et al. Improved progression free survival for patients with diabetes and locally advanced non-small cell lung cancer (NSCLC) using metformin during concurrent chemoradiotherapy. Radiother Oncol. 2016;118(3):453-459.

26. Jang WI, Kim MS, Lim JS, et al. Survival advantage associated with metformin usage in hepatocellular carcinoma patients receiving radiotherapy: a propensity score matching analysis. Anticancer Res. 2015;35(9):5047-5054.

27. Spratt DE, Zhang C, Zumsteg ZS, Pei X, Zhang Z, Zelefsky MJ. Metformin and prostate cancer: reduced development of castrationresistant disease and prostate cancer mortality. Eur Urol. 2013;63(4): 709-716.

28. Zannella VE, dal Pra A, Muaddi H, et al. Reprogramming metabolism with metformin improves tumor oxygenation and radiotherapy response. Clin Cancer Res. 2013;19(24):6741-6750.

29. Taira AV, Merrick GS, Galbreath RW, Morris M, Butler WM, Adamovich E. Metformin is not associated with improved biochemical free survival or cause-specific survival in men with prostate cancer treated with permanent interstitial brachytherapy. J Contemp Brachytherapy. 2014;6(3):254-261.

30. Skinner HD, Mccurdy MR, Echeverria AE, et al. Metformin use and improved response to therapy in esophageal adenocarcinoma. Acta Oncol. 2013;52(5):1002-1009.

31. Spierings LEAMM, Lagarde SM, van Oijen MGH, et al. Metformin use during treatment of potentially curable esophageal cancer patients is not associated with better outcomes. Ann Surg Oncol. 2015;22(S3):766-771.

32. van de Voorde L, Janssen L, Larue R, et al. Can metformin improve 'the tomorrow' of patients treated for oesophageal cancer? Eur J Surg Oncol. 2015;41(10):1333-1339.
33. Skinner HD, Sandulache VC, Ow TJ, et al. TP53 disruptive mutations lead to head and neck cancer treatment failure through inhibition of radiation-induced senescence. Clin Cancer Res. 2012;18(1):290-300.

34. Spratt DE, Beadle BM, Zumsteg ZS, et al. The influence of diabetes mellitus and metformin on distant metastases in oropharyngeal cancer: a multicenter study. Int J Radiat Oncol Biol Phys. 2016;94(3):523-531.

35. American Diabetes Association. Standards of medical care in diabetes-2016 abridged for primary care providers. Clin Diabetes. 2016;34(1): $3-21$.

36. Bojkova B, Orendas P, Garajova M, et al. Metformin in chemically-induced mammary carcinogenesis in rats. Neoplasma. 2009;56(3):269-274.

37. Gandini S, Puntoni M, Heckman-Stoddard BM, et al. Metformin and cancer risk and mortality: a systematic review and meta-analysis taking into account biases and confounders. Cancer Prev Res. 2014;7(9):867-885.

38. Levy A, Doyen J. Metformin for non-small cell lung cancer patients: opportunities and pitfalls. Crit Rev Oncol Hematol. 2018;125:41-47.

39. Cerezo M, Tomic T, Ballotti R, Rocchi S. Is it time to test biguanide metformin in the treatment of melanoma? Pigment Cell Melanoma Res. 2015;28(1):8-20.

40. Lin YC, Wu MH, Wei TT, et al. Metformin sensitizes anticancer effect of dasatinib in head and neck squamous cell carcinoma cells through AMPK-dependent ER stress. Oncotarget. 2014;5(1):298-308.

41. Rieken M, Xylinas E, Kluth L, et al. Association of diabetes mellitus and metformin use with oncological outcomes of patients with nonmuscle-invasive bladder cancer. BJU Int. 2013;112(8):1105-1112.

42. Zhang T, Zhang L, Zhang T, et al. Metformin sensitizes prostate cancer cells to radiation through EGFR/p-DNA-PKCS in vitro and in vivo. Radiat Res. 2014;181(6):641-649.

43. Kim EH, Kim MS, Cho CK, Jung WG, Jeong YK, Jeong JH. Low and high linear energy transfer radiation sensitization of HCC cells by metformin. J Radiat Res. 2014;55(3):432-442.

44. Vousden KH, Lane DP. p53 in health and disease. Nat Rev Mol Cell Biol. 2007;8(4):275-283.

45. Muller PA, Vousden KH. p53 mutations in cancer. Nat Cell Biol. 2013;15(1):2-8

46. Fasih A, Elbaz HA, Hüttemann M, Konski AA, Zielske SP. Radiosensitization of pancreatic cancer cells by metformin through the AMPK pathway. Radiat Res. 2014;182(1):50-59.
Cancer Management and Research

\section{Publish your work in this journal}

Cancer Management and Research is an international, peer-reviewed open access journal focusing on cancer research and the optimal use of preventative and integrated treatment interventions to achieve improved outcomes, enhanced survival and quality of life for the cancer patient. The manuscript management system is completely online and includes

\section{Dovepress}

a very quick and fair peer-review system, which is all easy to use. Visit $\mathrm{http}: / / \mathrm{www}$.dovepress.com/testimonials.php to read real quotes from published authors. 